\title{
Berberine alleviates cisplatin-induced acute kidney injury by regulating mitophagy via PINK 1/Parkin pathway
}

\author{
Jieying Qi ${ }^{1 \#}$, Qi Xue ${ }^{2 \#}$, Liuyan Kuang ${ }^{1}$, Lifen Xie ${ }^{1}$, Ren Luo ${ }^{1}$, Xiaoli Nie ${ }^{2}$ \\ ${ }^{1}$ School of Traditional Chinese Medicine, ${ }^{2}$ Hospital of Integrated Traditional Chinese Medicine and Western Medicine, Southern Medical University, \\ Guangzhou, China \\ Contributions: (I) Conception and design: J Qi, Q Xue, R Luo, X Nie; (II) Administrative support: L Kuang; (III) Provision of study materials or \\ patients: L Xie; (IV) Collection and assembly of data: Q Xue, L Kuang, L Xie; (V) Data analysis and interpretation: J Qi, Q Xue, R Luo, X Nie; (VI) \\ Manuscript writing: All authors; (VII) Final approval of manuscript: All authors. \\ \#The authors contributed equally to this work. \\ Correspondence to: Ren Luo. School of Traditional Chinese Medicine, Southern Medical University, Guangzhou 510515, China. Email: luoren@ \\ wo.com.cn; Xiaoli Nie. Hospital of Integrated Traditional Chinese Medicine and Western Medicine, Southern Medical University, Guangzhou \\ 510315, China. Email: nxl117@163.com.
}

Background: To study the protective effect of berberine (BBR) on cisplatin-induced acute kidney injury (AKI) and its effect on mitophagy.

Methods: (I) Male C57BL/6 mice aged 6-8 weeks were randomly divided into control group (saline), cisplatin group (cisplatin), and cisplatin + BBR $(5,10 \mathrm{mg} / \mathrm{kg})$ groups. In the cisplatin group and BBR groups, mice were injected intraperitoneally with $15 \mathrm{mg} / \mathrm{kg}$ of cisplatin. Mice in BBR groups were given BBR at 72, 48, 24, $0.5 \mathrm{~h}$ before and 24, $48 \mathrm{~h}$ after cisplatin injection. Mice were sacrificed $72 \mathrm{~h}$ after cisplatin injection, and blood were collected for detecting serum creatinine (SCr) and blood urea nitrogen (BUN) levels. Kidneys were collected for detecting protein expression levels of Kidney injury molecule 1 (KIM-1), LC3 II/LC3 I, p62, PINK 1, Parkin in the renal tissue by Western blotting. The pathological changes in renal tissues were observed using periodic acid-Schiff (PAS) staining. (II) Renal tubular epithelial cells (RTECs) were pretreated with different concentrations $(1,2$, and $4 \mu \mathrm{M})$ of BBR, and then incubated with cisplatin. Changes in autophagy proteins LC3 II/LC3 I, p62, PINK 1, and Parkin were detected by Western blotting, and changes in cellular reactive oxygen species (ROS) and mitochondrial membrane potential (MMP) were detected by flow cytometry.

Results: (I) Mice treated with BBR at dosage of 5 and $10 \mathrm{mg} / \mathrm{kg}$ for 6 days showed significant reduction in SCr and BUN compared to that in mice treated with cisplatin. PAS staining and immunohistochemistry showed that BBR ameliorated cisplatin-induced nephrotoxicity and reduced cisplatin-induced increase in protein expression levels of KIM-1. Compared to cisplatin-treated mice, the mice treated with BBR showed increased LC3 II/LC3 I, PINK 1, and Parkin, and decreased p62 protein expression. (II) Compared to cisplatin-incubated RTECs, cells pretreated with BBR for $24 \mathrm{~h}$ exhibited increased protein expressions of LC3 II/LC3 I, PINK1, and Parkin and decreased protein expression of p62. BBR reversed cellular ROS and cell MMP level induced by cisplatin.

Conclusions: BBR plays a protective role in cisplatin-induced AKI by up-regulating mitophagy in RTECs.

Keywords: Berberine (BBR); cisplatin; acute kidney injury (AKI); mitophagy; renal tubular epithelial cells (RTECs)

Submitted Jun 15, 2020. Accepted for publication Aug 12, 2020.

doi: $10.21037 /$ tau-20-1129

View this article at: http://dx.doi.org/10.21037/tau-20-1129 


\section{Introduction}

Acute kidney injury (AKI) is a clinical syndrome characterized by rapid decline in renal function caused by a variety of reasons (1), and its high morbidity and mortality makes it a global health problem (2). Cisplatin is an antitumor drug containing metal platinum and is widely used in the treatment of various solid tumors, such as gastric, non-small cell lung carcinoma. Renal toxicity is one of the most common side effects during cisplatin chemotherapy after a single dose of cisplatin $\left(50-100 \mathrm{mg} / \mathrm{m}^{2}\right)$, and about one-third of patients develop into AKI after cisplatin therapy (3-5). Proximal renal tubular injury is a characteristic pathophysiological mechanism of cisplatininduced AKI (6). However, there are no effective preventive and therapeutic measures for this type of AKI. Studying the mechanisms of cisplatin-induced AKI is of great significance for renal protection during tumor treatment.

Autophagy is the bulk degradation of cytosolic components, including aged or damaged proteins, other macromolecules, and organelles. Mitochondrial autophagy (mitophagy) is the selective engulfment of depolarized mitochondria, involving identification by PTEN-induced kinase 1 (PINK1) and ubiquitylation of OMM proteins by Parkin (7). The kidneys has high energy demands, and is sensitive to mitochondrial stress. Studies have indicated that autophagy, especially mitophagy, is a critical pathway for removal of damaged mitochondria and cellular repair after cell injury. Studies have shown that regulating mitophagy may be a key method to reducing cisplatin-induced AKI (8).

Berberine (BBR) is a quaternary ammonium isoquinoline alkaloid extract used in traditional Chinese medicine. Studies have found that BBR protects AKI caused by cisplatin by inhibiting oxidative stress and inflammation (9). However, it remains unclear whether BBR could regulate mitophagy. In this study, we aimed to evaluate the protective effects of BBR on cisplatin-induced AKI and the mechanisms on mitophagy of renal tubular epithelial cells (RTECs). We present the following article in accordance with the ARRIVE reporting checklist (available at http:// dx.doi.org/10.21037/tau-20-1129).

\section{Methods}

\section{Agents and materials}

Rat RTECs NRK-52E were purchased from Shanghai Zhongqiao Xinzhou Biology, and human RTECs (HKC cells) were donated by the team of Professor Zhou Lili from the National Clinical Research Center for Nephrology. Berberine (McLean, B875003), cisplatin (Sigma-Aldrich, P4394), Dulbecco's Modified Eagle Medium (DMEM) high-glucose medium (Gibco, NY, USA, C11995500BT), DMEM/F-12 basic medium (Gibco, NY, USA, C11330500BT), fetal bovine serum (FBS) (Gibco, NY, USA, A31608-02), trypsin (Gibco, NY, USA, 25200056), penicillin-streptomycin (Gibco, NY, USA, 15070063), beta actin antibody (Proteintech, IL , USA, 20536-1-AP), antiPINK1 antibody (Abcam, London, England, ab23707), anti-Parkin antibody (Abcam, London, England, ab77924), rabbit Kidney injury molecule 1 (KIM-1)/TIM-1 antibody (Novus, Colorado, USA, 26762), LC3A/B Rabbit mAb (CST, MA, USA, 12741), SQSTM1/p62 rabbit mAb (Abcam, London, England, ab109012), mitochondrial membrane potential (JC-1) detection kit (Beyotime, Shanghai, China, C2006), active oxygen detection kit (Beyotime, Shanghai, China, S0033), CCK8 kit (Dojindo, Kyushu, Japan, CK04), BCA protein quantification kit (Thermo, MA, USA, A53225), and 0.9\% saline (Kelun Pharmaceutical), were also obtained. Culture bottles (dish), centrifuge tubes, culture plates, and other consumables were all purchased from NEST company (Wuxi, China). This study was approved by the Laboratory Animal Ethics Committee of Nanfang Hospital (approval number: NFYY2019-104).

\section{Experimental animals}

A total of 32 specific pathogen-free (SPF) grade male C57BL/6J mice, age 6-8 weeks, with a body mass of $21 \pm 2 \mathrm{~g}$, were purchased from Speifo (Beijing) Biotechnology Co., Ltd. They were bred in the Laboratory Animal Center of Southern Hospital of Southern Medical University, at a temperature of $25.0 \pm 2.0^{\circ} \mathrm{C}$, alternating lighting every 12 hours, and given food and water ad libitum. The operation process of all experimental animals was strictly in accordance with the "Guide to the Care and Use of Experimental Animals" (2016). The mice were fed adaptively for 1 week and were fed a standard diet and water.

\section{Instruments}

A RS-20III cryogenic refrigerated centrifuge was purchased from Japan Tomy Seiko company (Japan); a Beckman AU4802 was purchased from Beckman Coulter (US); an upright optical microscope was purchased from Japan 
Nikon company (Japan); an embedding machine, freezing table, and tissue spreader were purchased from Wuhan Junjie Electronics Co., Ltd. (China); a protein electrophoresis instrument and protein transfer membrane instrument were purchased from Bio-Rad (USA).

\section{Study methods}

\section{Cisplatin-induced AKI mouse model}

Mice were randomly divided into control, cisplatin, BBR low-dose $(5 \mathrm{mg} / \mathrm{kg})$ group, and BBR high-dose $(10 \mathrm{mg} / \mathrm{kg})$ group. A single intraperitoneal injection of cisplatin at a dose of $15 \mathrm{mg} / \mathrm{kg}$ was used to establish a mouse AKI model. The BBR low-dose and high-dose group were given intraperitoneal injection of BBR solution 5 or $10 \mathrm{mg} / \mathrm{kg}$ at $72,48,24,0.5 \mathrm{~h}$ before and 24 and $48 \mathrm{~h}$ after cisplatin treatment. The mice in control group and cisplatin group were intraperitoneally injected with the same amount of normal saline. All mice were sacrificed $72 \mathrm{~h}$ after the last cisplatin treatment.

\section{Sample collection and processing}

After all mice were anesthetized, blood was collected through the celiac vein. Serum samples were obtained by centrifugation at 5,000 $\times \mathrm{g}$ for $10 \mathrm{~min}$. Kidney tissue was then quickly separated on an ice table, and $2 \mathrm{~mm}$ of kidney tissue were cut horizontally and fixed with $4 \%$ paraformaldehyde solution at $4{ }^{\circ} \mathrm{C}$ for $24 \mathrm{~h}$. Specimens were dehydrated and embedded for storage. The remaining kidney tissue samples and plasma samples were stored in the refrigerator at $-80^{\circ} \mathrm{C}$ for future use.

\section{Detection of serum biochemical indicators}

The levels of serum creatinine (SCr) and blood urea nitrogen (BUN) were detected by an automatic biochemical analyzer.

\section{Periodic acid-Schiff (PAS) staining of kidney sample}

After the kidney tissue was fixed with $4 \%$ paraformaldehyde for $24 \mathrm{~h}$, it was dehydrated and embedded in paraffin. A $4 \mu \mathrm{m}$ thick paraffin section was cut and stained with PAS. The degree of tissue damage was observed under an optical microscope, and the renal tubular injury score was recorded. Ten fields of high-magnification in microscope were randomly selected from each tissue for scoring. Score was calculated according to the following criteria: 0 points, no obvious injury; 1 point, mild injury, presence of epithelial cell swelling, and luminal expansion; 2 points, severe injury, renal tubular epithelial cell nuclear staining missing with the lumen of the renal tubule blocked; 3 points, damaged renal tubule epithelial cells, with cell shedding, disappearance of nuclear staining, and a large number of casts. A doubleblind random analysis was used. The average value of 10 visual fields for each film was evaluated and analyzed.

\section{Cell culture and treatment}

Rat RTECs NRK-52E were cultured in DMEM highglucose medium containing 10\% FBS and 1\% penicillinstreptomycin. Human RTECs HKC were cultured with DMEM/F-12 medium containing $10 \%$ FBS and $1 \%$ penicillin-streptomycin. All cells were cultured in an incubator at $37{ }^{\circ} \mathrm{C}, 5 \% \mathrm{CO}_{2}$, and $90 \%$ relative humidity. The logarithmic phase cells were selected for experiments. The cells were divided into control group, cisplatin group, and BBR-low, BBR-medium, BBR-high groups with different concentrations of BBR $(1,2$, and $4 \mu M)$.

\section{CCK8 detection of cell proliferation}

NRK-52E and HKC cells in the logarithmic phase were seeded on 96-well culture plate and the cells grow to approximately $70 \sim 80 \%$ confluence in complete medium for 12 hours. Then cultures were supplemented with different concentrations of BBR $(1,2$, and $4 \mu M)$ for 12 hours. Next, the cisplatin group and BBR group cells were treated with $50 \mu \mathrm{M}$ cisplatin for 12 hours. Cell viability was measured by CCK 8 assay. Then, $10 \mu \mathrm{L}$ of CCK8 solution were added to each well, and incubated for 3 hours. Finally, the absorbance at $450 \mathrm{~nm}$ was measured with a microplate reader.

\section{Western blot detection of target protein expression in renal tissues and cells}

Mouse kidney tissue was taken out from a $-80{ }^{\circ} \mathrm{C}$ refrigerator and weighed. Radio immunoprecipitation assay (RIPA) lysate was added to the specimen and ground with a tissue grinder. Tissue lysate was collected and centrifuged, and the supernatant was collected. The NRK-52E and HKC cells in the logarithmic phase were collected and seeded into 6 -well plates at $3 \times 10^{5}$ cells/well. Next, $2 \mathrm{~mL}$ of fresh medium was added. After cells grow to approximately $70-80 \%$ confluence, they were incubated with BBR with different concentrations for 12 hours. The cisplatin group and the BBR group were treated with $50 \mu \mathrm{M}$ of cisplatin for $12 \mathrm{~h}$. The cells were washed 3 times with prechilled phosphate-buffered saline (PBS) and lysed with RIPA. The bicinchoninic acid (BCA) method was used to determine 
A

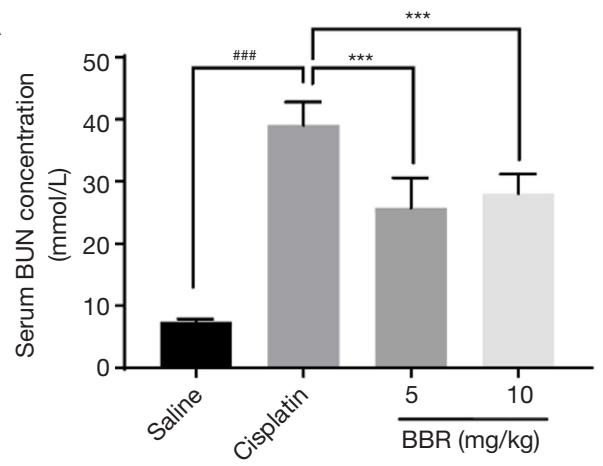

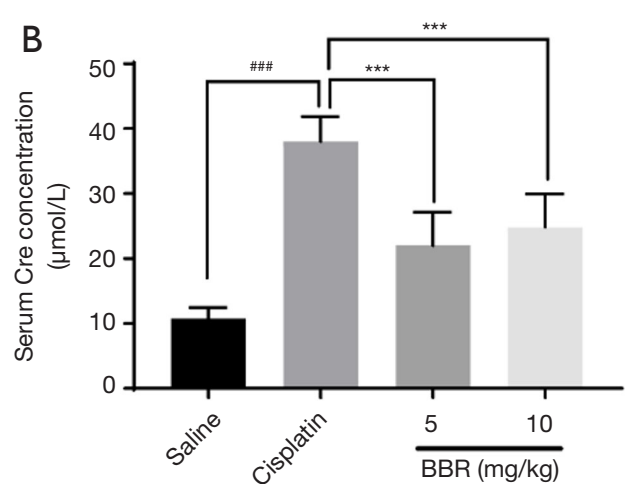

Figure 1 Serum BUN (A) and SCr (B) levels in mice. ${ }^{\# \# \# ~} \mathrm{P}<0.001$ vs. saline group (control); ${ }^{* * *} \mathrm{P}<0.001$ vs. cisplatin group. BBR, berberine; BUN, blood urea nitrogen; $\mathrm{SCr}$, serum creatinine.

the protein concentration of the sample, with $5 \times$ loading buffer being added to the specimen and boiled at $98{ }^{\circ} \mathrm{C}$ for $5 \mathrm{~min}$. Then, $50 \mu \mathrm{g}$ of sample per well was added, and the protein was separated by SDS-PAGE $(15 \%$ or $12 \%)$ and transferred onto polyvinylidene fluoride (PVDF) membrane at a constant current of $320 \mathrm{~mA}$ for $120 \mathrm{~min}$. After blocking with $5 \%$ BSA for 2 hours, membranes were incubated with the primary antibodies overnight at $4^{\circ} \mathrm{C}$. After washing with TBST for three times, the membranes were incubated with the secondary antibodies for 2 hours at room temperature, followed by enhanced chemiluminescence reaction. The protein bands were measured using Image J software.

\section{Determination of cell reactive oxygen specie $s$ (ROS)}

The NRK-52E and HKC cells in the logarithmic phase were seeded into 6 -well plates at $3 \times 10^{5}$ cells/well. Then $2 \mathrm{~mL}$ of fresh medium was added to each well. After cells grow to approximately $70-80 \%$ confluence, BBR with different concentrations was added for pretreatment for 12 hours. Next, the cisplatin group and BBR groups were treated with $50 \mu \mathrm{M}$ cisplatin for 12 hours. Then cell culture medium was removed, and cells were incubated at $37{ }^{\circ} \mathrm{C}$ for $20 \mathrm{~min}$ with dichlorodihydrofluorescein-acetoacetate (DCFH-DA) (10 $\mu \mathrm{M}, 1.2 \mathrm{~mL} /$ well). Then cells washed, resuspended, and detected by flow cytometry.

\section{Measurement of cell mitochondrial membrane potential}

The NRK-52E and HKC cells in the logarithmic phase were seeded into 6 -well plates at $3 \times 10^{5}$ cells/well. Then, $2 \mathrm{~mL}$ of fresh medium was added to each well. After cells grow to approximately $70-80 \%$ confluence, BBR with different concentrations was added for pretreatment for
12 hours. The cisplatin group and the BBR groups were treated with $50 \mu \mathrm{M}$ of cisplatin for 12 hours. JC-1 fluorescent probe was loaded and cells were incubated at $37^{\circ} \mathrm{C}$ for $20 \mathrm{~min}$. After washing cells with staining buffer 3 times, cells were resuspended, and detected by flow cytometry.

\section{Statistical analysis}

All experiments were independently repeated 3 times. GraphPad Prism 7 was used for statistical analysis and graphing. All data are expressed as mean $\pm \mathrm{SD}$. The mean value of two samples was compared using independent samples $t$ test. Multiple groups were compared by performing one-way ANOVA following Tukey multiple comparisons tests. $\mathrm{P}$ value $<0.05$ was considered statistically significant.

\section{Results}

\section{Effect of BBR on serum BUN and SCr in mice}

The results showed that the BUN and SCr levels in the cisplatin group were significantly higher than those in the control group $(\mathrm{P}<0.001)$, while the $\mathrm{BUN}$ and $\mathrm{SCr}$ in mice of BBR groups were significantly lower than those in the cisplatin group $(\mathrm{P}<0.001)$ (Figure 1).

\section{The effect of BBR on the histopathology in mouse kidneys}

PAS staining showed that, compared to the control group, mice in the cisplatin group had significant damage in renal tubular epithelial cells, including cell swelling, necrosis, and 

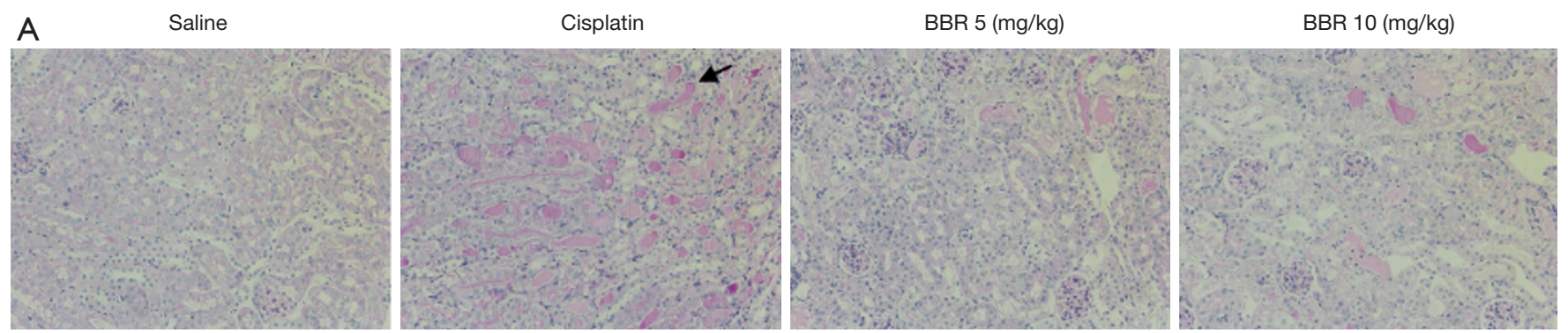

B

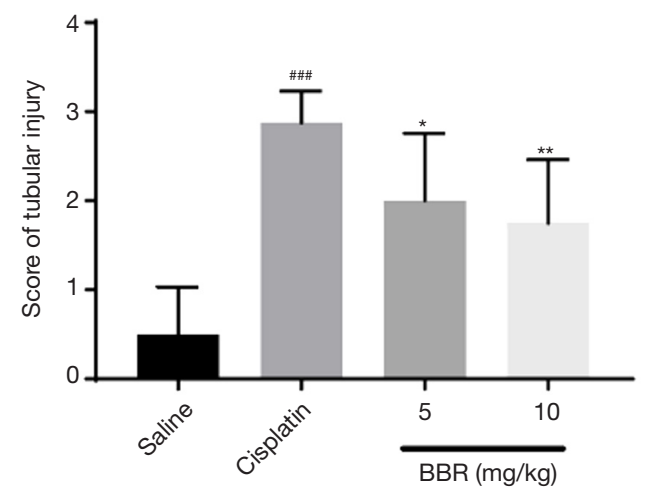

Figure 2 Histopathological result of kidney tissues in mice. (A) Pathological results of kidney tissues (PAS, $\times 200)$; the black arrow indicates the tubular type. (B) Renal tubular injury score. ${ }^{\# \# \#} \mathrm{P}<0.001$ vs. saline group; ${ }^{*} \mathrm{P}<0.05,{ }^{* *} \mathrm{P}<0.01$ vs. cisplatin group. BBR, berberine; $\mathrm{PAS}$, periodic acid-Schiff staining.

shedding, cell nuclear staining disappeared, a large number of tube cast appeared, and there was more inflammatory cell infiltration in the renal tubule interstitium. In mice of BBR groups, renal tubular injury was significantly reduced, and the morphological structure was close to that of the control group (Figure 2). These results suggest that BBR alleviates kidney damage caused by cisplatin.

\section{The effect of BBR on the protein expression of KIM-1 in mouse kidneys}

KIM-1 is an important marker of renal tubular injury (10). Renal tissue immunohistochemistry indicated that a large number of KIM-1 positive-staining antigens could be seen in the renal tubular cell membrane, cytoplasm, and lumen in the renal cortex and marrow junction in the cisplatin group mice. The number of KIM-1 positive renal tubules in renal tissue of BBR low-dose group $(\mathrm{P}<0.05)$ and high-dose group $(\mathrm{P}<0.01)$ was significantly reduced (Figure $3 A, B$ ). Western blotting results showed that, compared to the control group, the KIM-1 protein expression level in the kidney of the cisplatin group mice was significantly increased $(\mathrm{P}<0.01)$. The protein expression level of KIM-1 in kidney tissue of BBR low-dose group $(\mathrm{P}<0.05)$ and high-dose group $(\mathrm{P}<0.001)$ was significantly lower than that of the cisplatin group (Figure $3 C, D)$.

\section{The effect of BBR on the expression of autophagy-related proteins LC3 II/LC3 I, p62, PINK 1 and Parkin in mouse kidneys}

Western blotting results showed that compared to the control group, the protein expression levels of autophagyrelated proteins LC3 II/LC3 I $(\mathrm{P}<0.01)$, PINK $1(\mathrm{P}<0.05)$, and Parkin $(\mathrm{P}<0.05)$ in the mice of cisplatin group were significantly decreased, while the protein expression level of p62 was significantly increased $(\mathrm{P}<0.05)$. BBR intervention significantly upregulated the protein expression levels of LC3 II/LC3 I, PINK 1 and Parkin, and downregulated the protein expression level of p62 $(\mathrm{P}<0.05)$ (Figure 4).

\section{The effect of BBR on cell viability}

The results showed that compared to the control group, cisplatin caused a significant decrease in the cell viability 

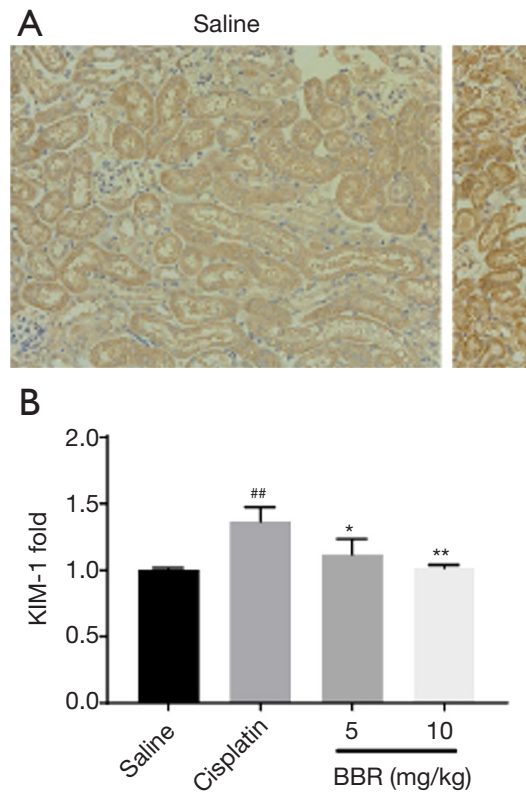

Cisplatin

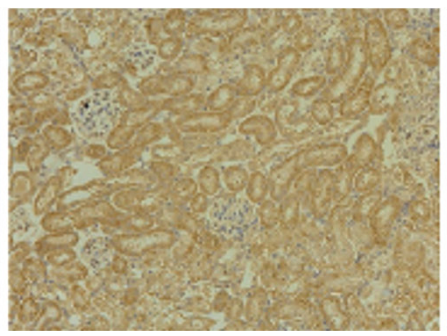

C
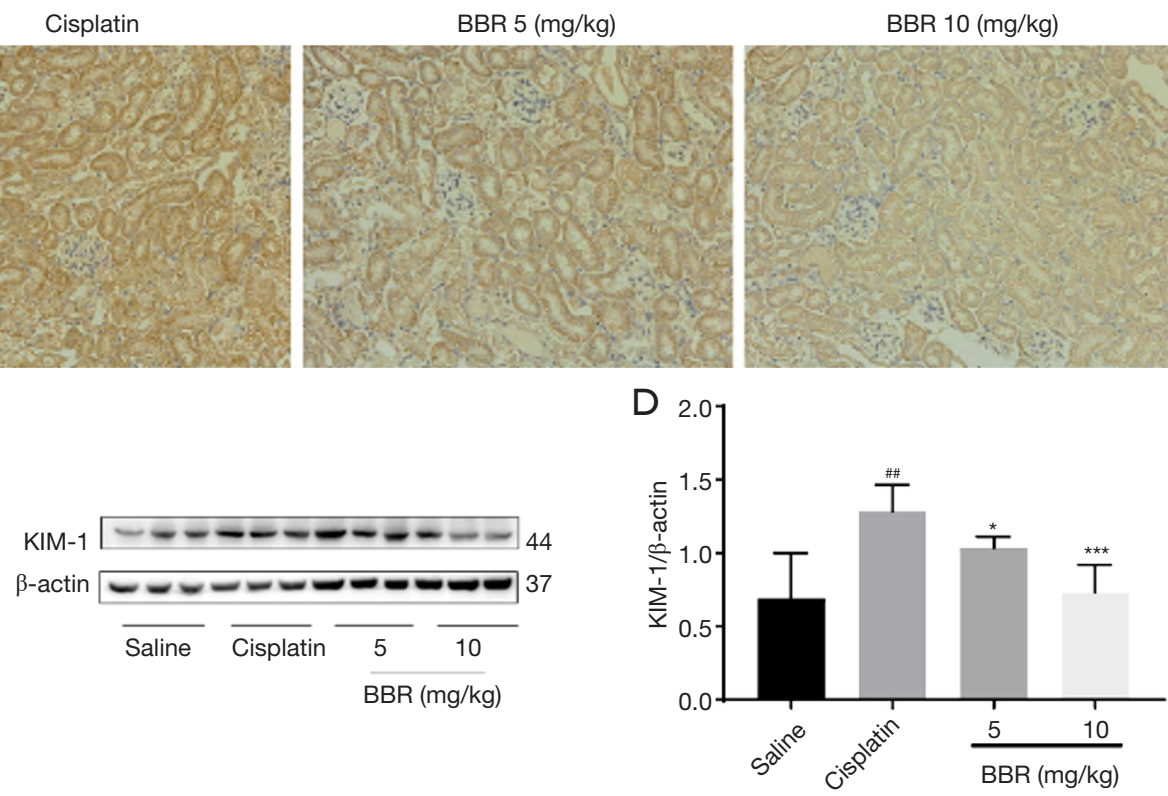

Figure 3 KIM-1 protein expression level in kidney tissues in mice. (A) Immunohistochemistry and (B) quantitative data of KIM-1 protein; (C) Western blotting analysis and (D) quantitative analysis of $\mathrm{KIM}-1$ protein. ${ }^{*} \mathrm{P}<0.01$ vs. saline group; ${ }^{* *} \mathrm{P}<0.05$ vs. saline group; ${ }^{*} \mathrm{P}<0.05$, *** $\mathrm{P}<0.001$ vs. cisplatin group. BBR, berberine; KIM-1, Kidney injury molecule 1 .

of NRK-52E cells $(\mathrm{P}<0.001)$ and HKC cells $(\mathrm{P}<0.001)$. Meanwhile, compared to the cisplatin group, BBR at different dosage $(1,2,4 \mu \mathrm{M})$ significantly increased cell viability compared to cisplatin treatment (Figure 5).

\section{The effect of BBR on cell autophagy}

Western blotting results showed that, compared to the control group, the autophagy marker proteins LC3 II/LC3 $\mathrm{I}$ in NRK-52E cells $(\mathrm{P}<0.01)$ and HKC cells $(\mathrm{P}<0.05)$ were decreased to basal levels. Compared to the cisplatin group, BBR significantly increased the ratio of LC3II/LC3I and degradation of p62 (Figure 6), and significantly upregulated the protein expressions of PINK1 and Parkin (Figure 7).

\section{Effect of BBR on intracellular ROS levels}

The results of flow cytometry showed that compared to the control group, the intracellular ROS production in the cisplatin group cells were significantly increased $(\mathrm{P}<0.01)$. BBR significantly reduced the production of ROS in RTECs (Figure 8).

\section{The effect of BBR on cell mitochondrial membrane potential}

The results of flow cytometry showed that compared to the control group, the mitochondrial membrane potential of NRK-52E cells $(\mathrm{P}<0.001)$ and HKC cells $(\mathrm{P}<0.01)$ in the cisplatin group decreased significantly. The intervention of BBR significantly increased the mitochondrial membrane potential (Figure 9).

\section{Discussion}

Cisplatin is one of the most widely used and effective chemotherapy drugs (11); however, its application and efficacy in cancer treatment has been restricted because of its nephrotoxicity. One of the pathophysiological mechanisms of cisplatin-induced AKI is proximal tubular injury. Clinically, cisplatin treatment is often used in combination with short-term, low-dose, and low-volume of hydration regimens to reduce the side effects (4). Therefore, drugs that can effectively prevent renal tubular cell damage and AKI have important clinical significance. BBR is an isoquinoline alkaloid extracted from Coptis chinensis, 

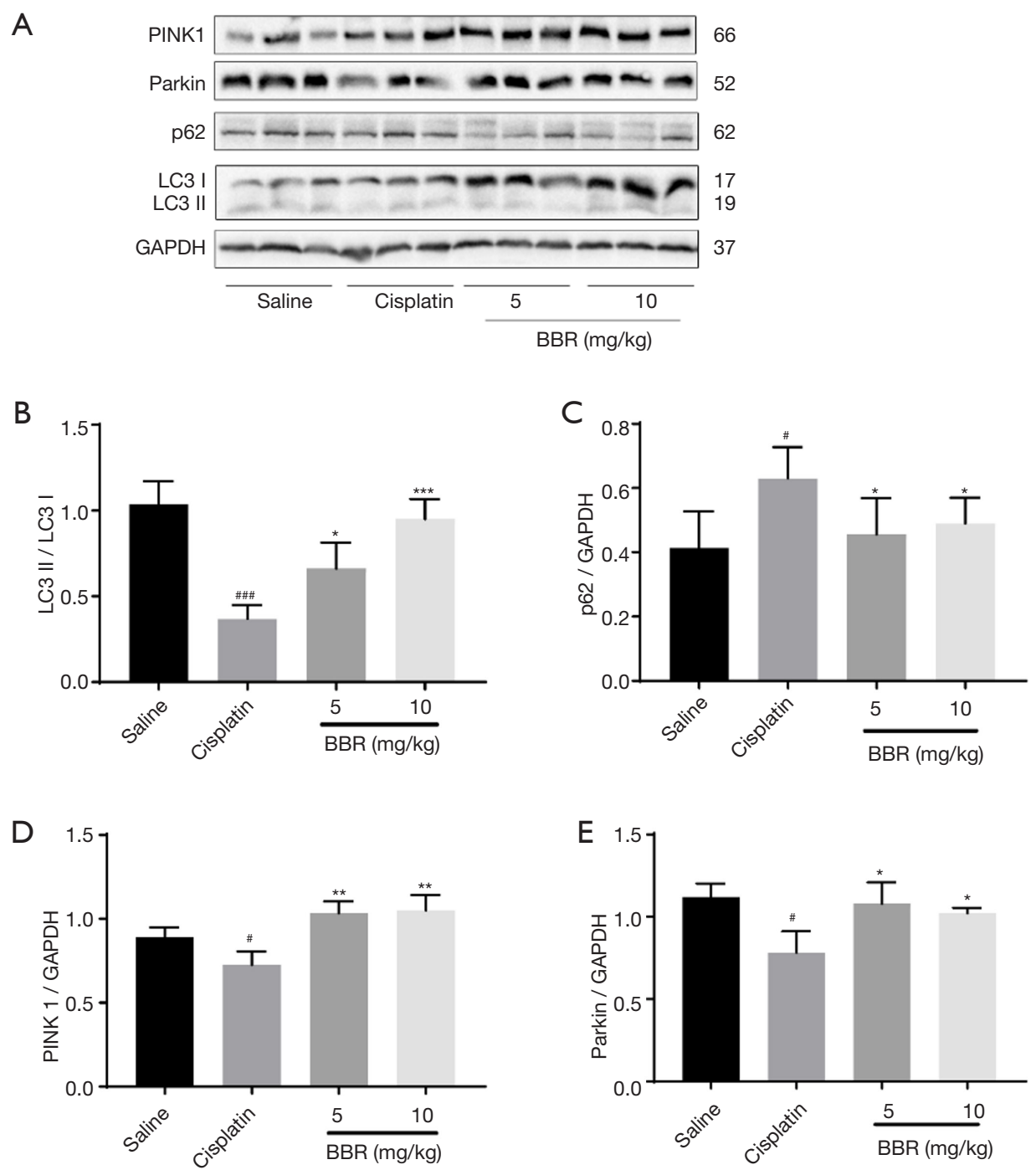

Figure 4 Kidney autophagy in mice with cisplatin-induced AKI. (A) Representative blots of LC3 II/LC3 I, p62, PINK, and Parkin in kidney tissues by Western blotting. (B,C,D,E) LC3 II/LC3 I, p62, PINK 1, and Parkin ratio were subjected to semi-quantitative analysis by Image J software. ${ }^{\#} \mathrm{P}<0.05,{ }^{\# \# \#} \mathrm{P}<0.001$ vs. saline group; ${ }^{*} \mathrm{P}<0.05,{ }^{* *} \mathrm{P}<0.01,{ }^{* *} \mathrm{P}<0.001$ vs. cisplatin group. BBR, berberine; AKI, acute kidney injury.

Phellodendron amurense, rhubarb and other plants. Studies have shown that BBR can regulate immunity, promote antiinflammatory factors, and reduce endoplasmic reticulum stress (12). Through in vivo models of cisplatin-induced AKI, this study confirmed that cisplatin caused a significant decrease in renal function and renal tubular damage in mice. After BBR intervention, serum BUN and SCr levels were decreased, renal tubular injury damage was alleviated, and the expression of renal tubular injury marker KIM-1 was downregulated. These results suggest that BBR plays a protective role in AKI caused by cisplatin.
Evolutionarily, autophagy is a highly conserved molecular mechanism. Damaged organelles, protein multimers, and other large molecules in the cytoplasm are isolated into autophagic vesicles and then delivered to lysosomes for degradation (13). Mitochondria are important sites for the production of eukaryotic adenosine triphosphate (ATP) and act as the cells' energy factory. Mitophagy is a type of autophagy, a process by which cells selectively remove damaged or dysfunctional mitochondria, thereby maintaining the homeostasis of the intracellular environment (14). Tubular epithelial cells are rich in 
A

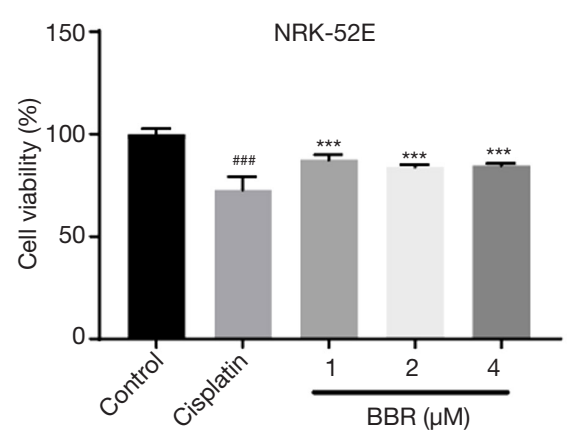

B

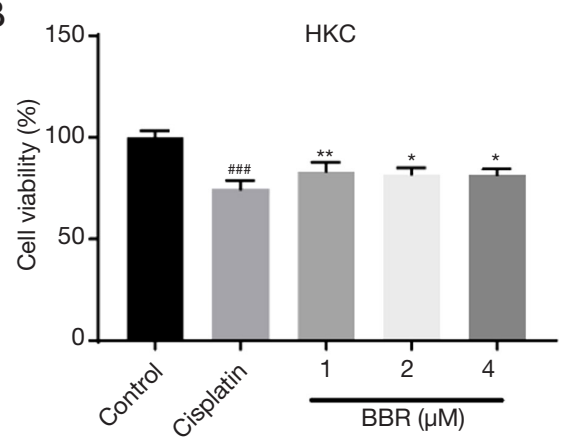

Figure 5 BBR alleviated cisplatin-induced low cell viability. (A) NRK-52E and (B) HKC cell viability were tested by CCK8 assays. ${ }^{\# \# !} \mathrm{P}<0.001$ vs. control group; ${ }^{*} \mathrm{P}<0.05,{ }^{* *} \mathrm{P}<0.01,{ }^{* * *} \mathrm{P}<0.001$ vs. cisplatin group. BBR, berberine.

A

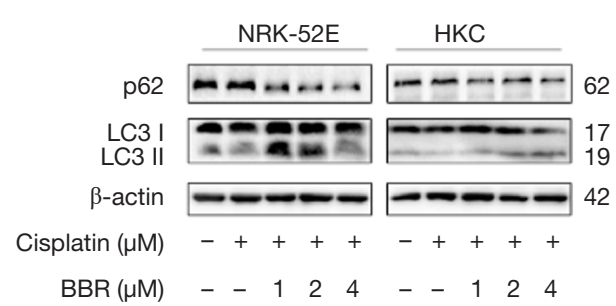

B

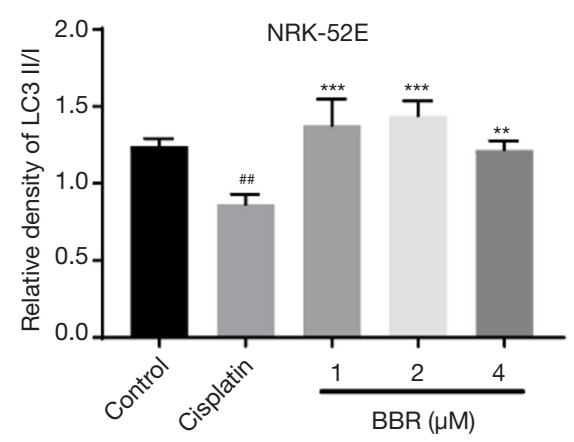

D

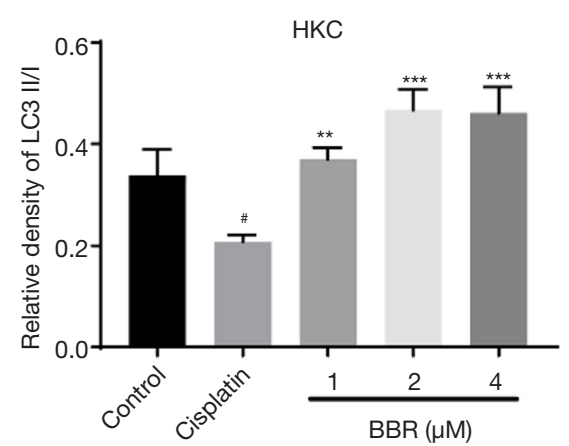

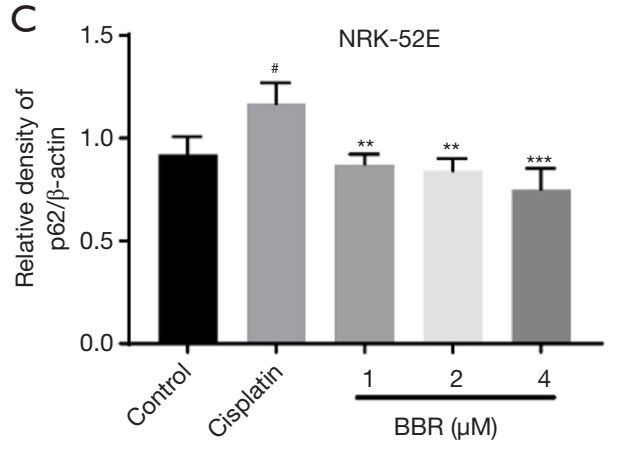

E

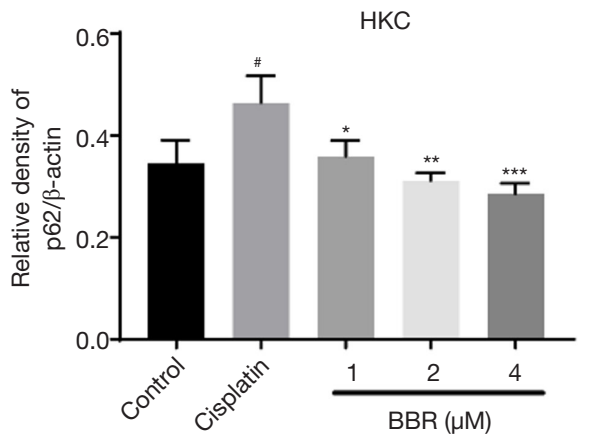

Figure 6 Cell autophagy protein expression level. (A) Representative blots of LC3 and p62 in NRK-52E cells and HKC cells. (B,C,D,E) Quantification of endogenous $\mathrm{LC} 3$ and $\mathrm{p} 62 .{ }^{\#} \mathrm{P}<0.05,{ }^{* \prime} \mathrm{P}<0.01$ vs. control group; ${ }^{*} \mathrm{P}<0.05,{ }^{* *} \mathrm{P}<0.01,{ }^{* * *} \mathrm{P}<0.001$ vs. cisplatin group. 
A

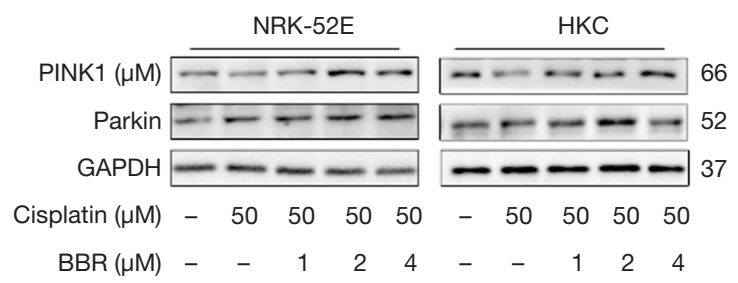

B

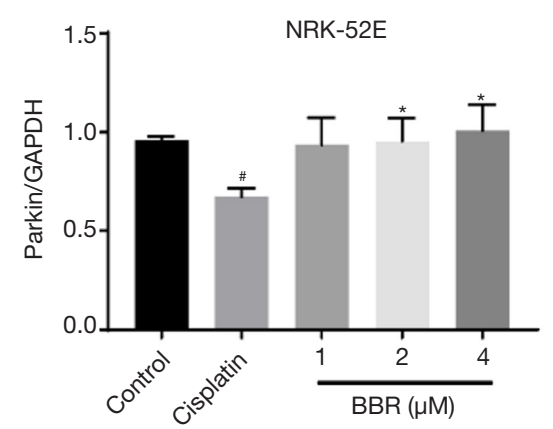

D

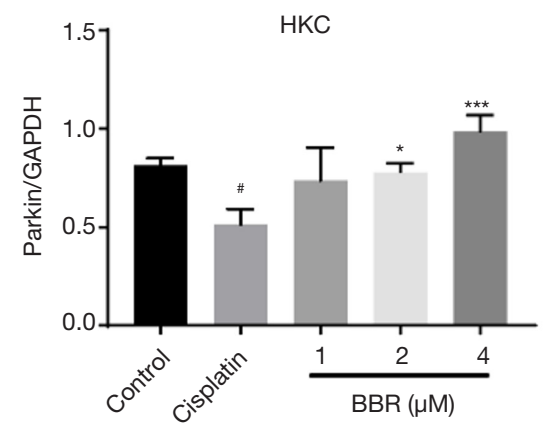

C

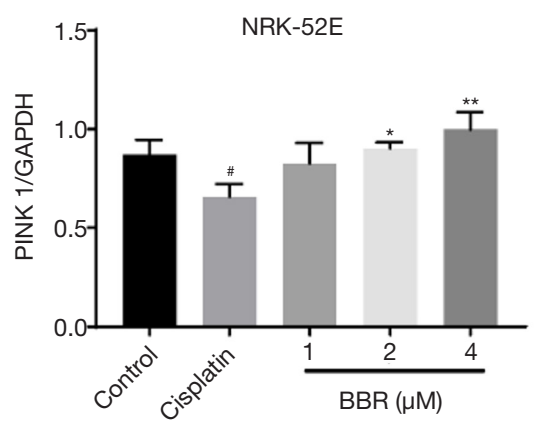

E

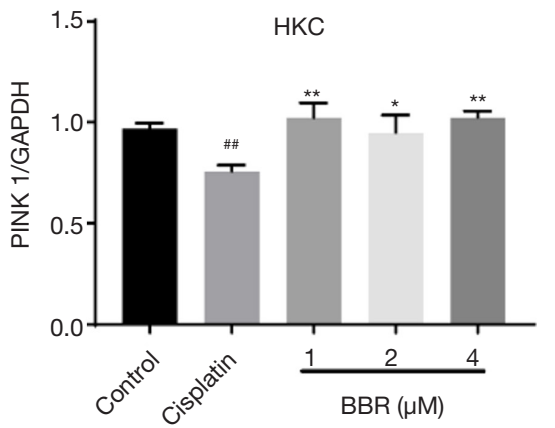

Figure 7 Expression of PINK 1 and Parkin in NRK-52E and HKC cells. (A) Representative blots of PINK 1 and Parkin in NRK-52E cells and HKC cells. (B,C,D,E) Bar graphs showing the quantification of PINK 1 and Parkin in NRK-52E cells and HKC cells. ${ }^{\#} \mathrm{P}<0.05,{ }^{\# \#} \mathrm{P}<0.01$ vs. control group; ${ }^{*} \mathrm{P}<0.05,{ }^{* *} \mathrm{P}<0.01,{ }^{* * *} \mathrm{P}<0.001$ vs. cisplatin group.

mitochondria. The secretory reabsorption function of renal tubules relies on mitochondrial oxidative phosphorylation to provide energy (15). Oxidative stress and inflammation during AKI promote mitochondrial depolarization and dysfunction (16), triggering mitophagy. Cells ensure the quality of mitochondria through mitophagy, and remove damaged mitochondria, thereby reducing the excessive release of ROS and pro-apoptotic substances caused by damaged mitochondria (17). PINK1/Parkin-mediated mitophagy pathway is one of the best studied mechanisms for mitophagy in mammalian cells (14). When autophagy occurs, LC3 I is activated by Atg7, undergoes ubiquitinlike processing and modification, and combines with phosphatidylethanolamine (PE) on the surface of the autophagy membrane to form LC3 II. LC3 II binds and is always located on the intracellular autophagosome membrane. Therefore, the ratio of LC3 II to LC3 I can be regarded as a marker of autophagy (18), and the amount of LC3 II reflects the number of autophagosomes (19). In the late stage of autophagy, LC3 II is degraded by SQSTM1/ p62, and p62 binds to ubiquitinated protein and form a complex with LC3, so that the protein is transferred into autophagy lysosome for degradation. When autophagy occurs as the protein continues to degrade, p62 levels gradually decrease, and thus p62 is a marker protein to detect autophagy activity $(20,21)$.

Multiple studies have shown that BBR regulates the level of cellular autophagy. BBR inhibits basic autophagy in adipocytes and adipose tissue of mice fed with high-fat diet by downregulating the expression of BECLIN1 (22). In terms of atherosclerotic diseases, BBR inhibits inflammation of J774A.1 cells by inducing autophagy (23). 

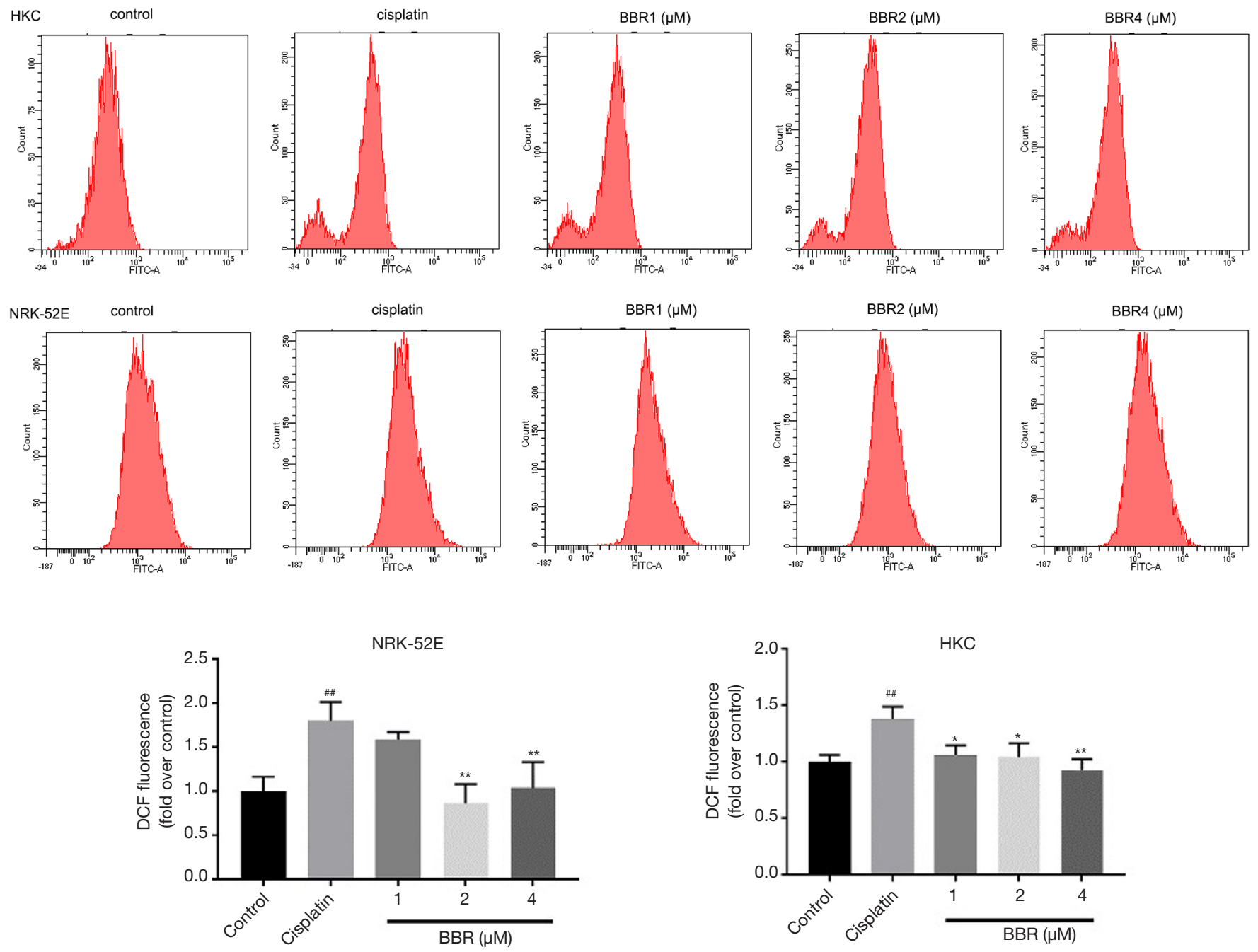

Figure 8 Cellular ROS production level in NRK-52E cells and HKC cells. ${ }^{\# \#} \mathrm{P}<0.01$ vs. control group; ${ }^{*} \mathrm{P}<0.05,{ }^{* *} \mathrm{P}<0.01$ vs. cisplatin group. ROS, reactive oxygen species.

In kidney disease, BBR inhibits renal oxidative stress and inflammatory response in diabetic mice, and activates the mitochondrial energy homeostasis and fatty acids of podocytes by activating the oxidative proliferatoractivated receptor $\gamma$ coactivator- $1 \alpha$ (PGC-1 $\alpha$ ) (24). BBR ameliorates diabetic nephropathy by inhibiting glomerular mesangial matrix expansion and activating autophagy (25), and alleviates palmitic acid-induced podocyte damage by inhibiting Drp1-mediated mitochondrial dysfunction (26). Using in vivo and in vitro models, this study found that cisplatin caused a decline in the protein expression levels of LC3 II/LC3 I, an increase in the level of p62, and a decrease in the levels of PINK 1/Parkin protein in kidney tissues and RTECs. After BBR intervention, LC3 II /LC3 I and PINK 1/Parkin protein levels were increased in kidney tissue, while p62 was decreased. These findings suggest that mitophagy may play an important role in the protective effects of BBR in cisplatin-induced AKI.

The question remains as to the mechanism by which BBR increases mitophagy. ROS, as a by-product of mitochondrial metabolism, has a dual effect on cells. Low levels of ROS induce mitophagy and other cell survival processes (27). However, high levels of ROS in cells directly or indirectly participate in cell signaling and induce apoptosis. Therefore, ROS is an important marker of mitochondrial dysfunction and injury. The decrease of mitochondrial membrane potential is a landmark event in the early stage of apoptosis. This study examined changes in intracellular ROS levels 
NRK-52E
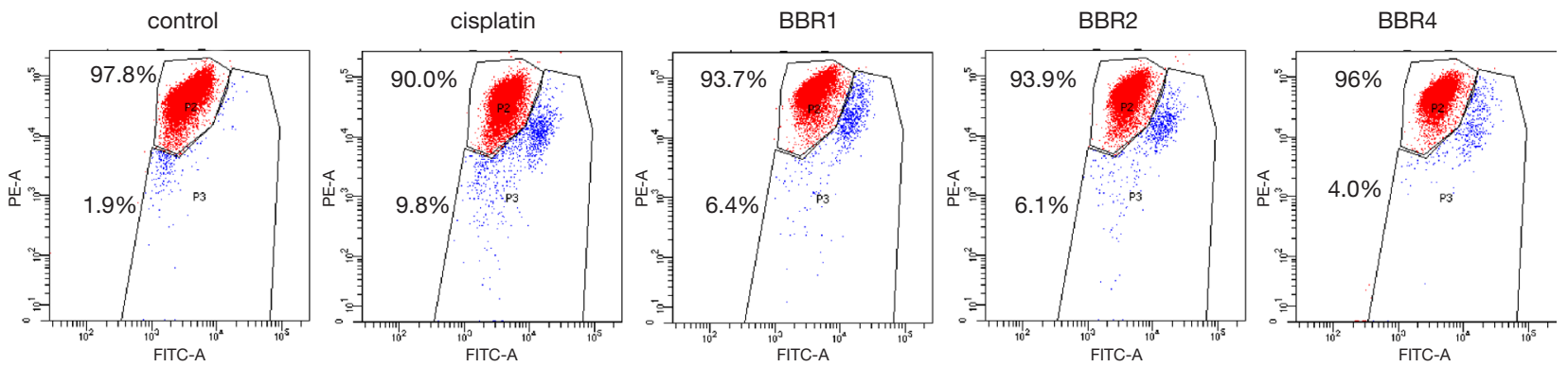

HKC

control

cisplatin

BBR1

BBR2

BBR4
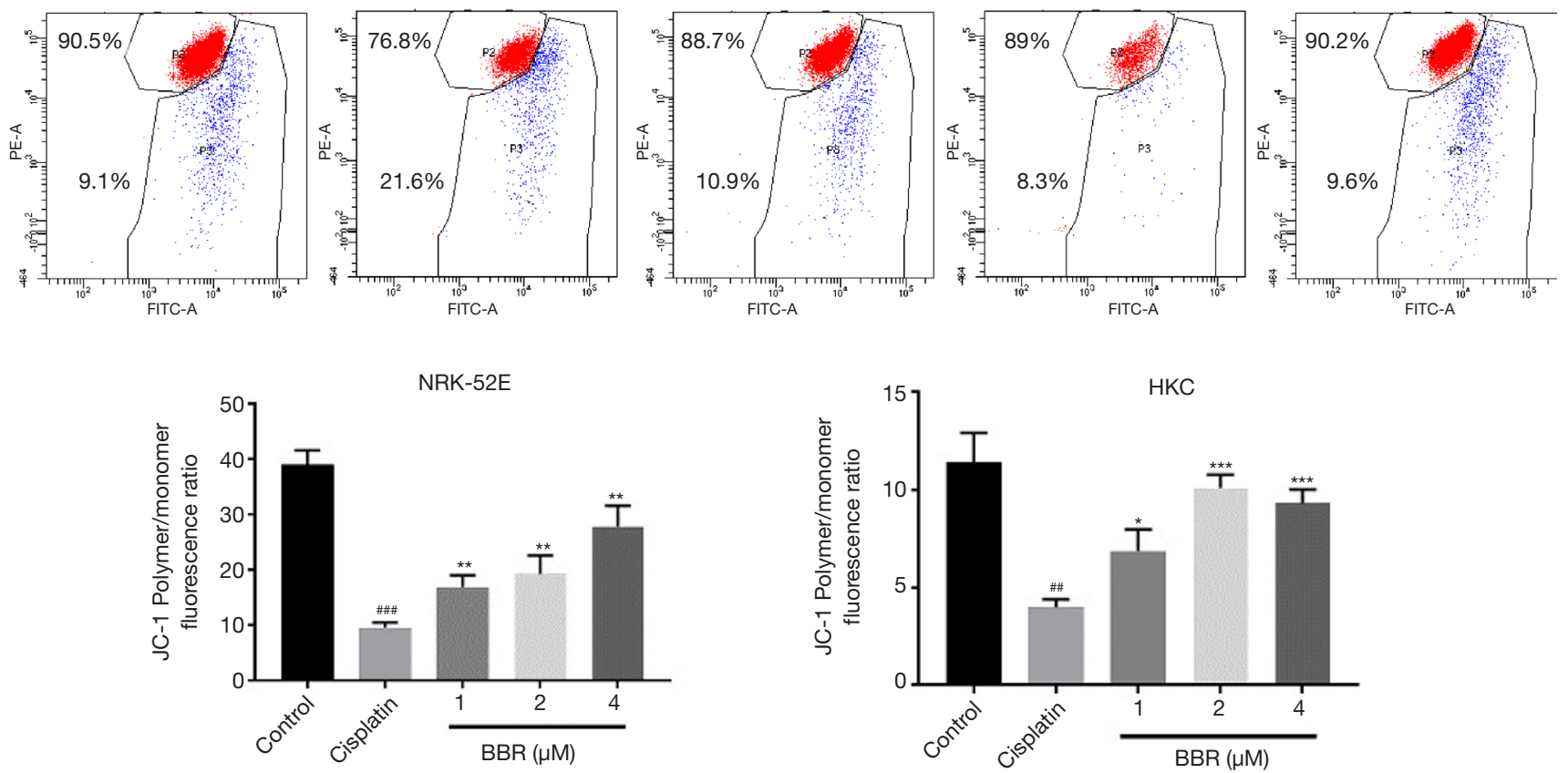

Figure 9 Cell MMP level in NRK-52E cells and HKC cells. ${ }^{\# \#} \mathrm{P}<0.05$ vs. control; ${ }^{\# \# \#} \mathrm{P}<0.001$ vs. control group; ${ }^{*} \mathrm{P}<0.05,{ }^{* *} \mathrm{P}<0.01$, ${ }^{* * *} \mathrm{P}<0.001 v s$. cisplatin group. MMP, mitochondrial membrane potential.

and changes in mitochondrial membrane potential in a cell model of cisplatin-induced renal tubular injury. The results showed that BBR reduced the production of ROS in RTECs and alleviated mitochondria membrane potential drops caused by cisplatin.

In this study, we elucidated that mitophagy triggered by BBR was mediated by activation of the PINK1/Parkin signal pathway in mice and RTECs. Mitophagy is a key pathway of cisplatin-induced nephrotoxicity, and the activation of mitophagy via PINK1/Parkin by BBR may antagonize the damaging effect through reduced accumulation of ROS.

In conclusion, we have demonstrated that BBR alleviates cisplatin-induced cytotoxicity in renal tubular cells and restores kidney function in a mice model. BBR could be viable therapeutic agents or adjuvants which reduce nephrotoxicity during cisplatin chemotherapy.

\section{Acknowledgments}

Funding: The National Natural Science Foundation of China (no. 81774038), the Guangdong Province Natural Science Foundation (no. 2015A030313300, 2016A030313538, and 2020A1515010849), the Guangdong Science and Technology Program (no. 2014A020221073); the Innovation Team of Chronic Kidney disease with Integrated Traditional Chinese and Western Medicine (no. 2019KCXTD014), the Clinical Research Project on National Inheritance and Innovation of Traditional 
Chinese Medicine, (no. 202006002), the Hospital of Integrated Traditional Chinese Medicine, Southern Medical University.

\section{Footnote}

Reporting Checklist: The authors have completed the ARRIVE reporting checklist. Available at http://dx.doi. org/10.21037/tau-20-1129

Data Sharing Statement: Available at http://dx.doi. org/10.21037/tau-20-1129

Conflicts of Interest: All authors have completed the ICMJE uniform disclosure form (available at http://dx.doi. org/10.21037/tau-20-1129). The authors have no conflicts of interest to declare.

Ethical Statement: The authors are accountable for all aspects of the work in ensuring that questions related to the accuracy or integrity of any part of the work are appropriately investigated and resolved. This study was approved by the Laboratory Animal Ethics Committee of Nanfang Hospital (approval number: NFYY-2019-104). The operation process of all experimental animals was strictly in accordance with the "Guide to the Care and Use of Experimental Animals" (2016).

Open Access Statement: This is an Open Access article distributed in accordance with the Creative Commons Attribution-NonCommercial-NoDerivs 4.0 International License (CC BY-NC-ND 4.0), which permits the noncommercial replication and distribution of the article with the strict proviso that no changes or edits are made and the original work is properly cited (including links to both the formal publication through the relevant DOI and the license). See: https://creativecommons.org/licenses/by-nc-nd/4.0/.

\section{References}

1. Moore PK, Hsu RK, Liu KD. Management of Acute Kidney Injury: Core Curriculum 2018. Am J Kidney Dis 2018;72:136-48.

2. Quenot JP, Dargent A, Large A, et al. Treatment of sepsisinduced acute kidney injury in the ICU: the therapeutic targets do not seem to be established yet. Ann Transl Med 2019;7:S181.
3. Lebwohl D, Canetta R. Clinical development of platinum complexes in cancer therapy: an historical perspective and an update. Eur J Cancer 1998;34:1522-34.

4. Crona DJ, Faso A, Nishijima TF, et al. A Systematic Review of Strategies to Prevent Cisplatin-Induced Nephrotoxicity. Oncologist 2017;22:609-19.

5. Latcha S, Jaimes E A, Patil S, et al. Long-Term Renal Outcomes after Cisplatin Treatment. Clin J Am Soc Nephrol 2016;11:1173-9.

6. Ozkok A, Edelstein C L. Pathophysiology of cisplatin-induced acute kidney injury. Biomed Res Int 2014;2014:967826.

7. Kaushal GP, Shah SV. Autophagy in acute kidney injury. Kidney Int 2016;89:779-91.

8. Wang Y, Tang C, Cai J, et al. PINK1/Parkin-mediated mitophagy is activated in cisplatin nephrotoxicity to protect against kidney injury. Cell Death Dis 2018;9:1113.

9. Domitrović R, Cvijanović O, Pernjak-Pugel E, et al. Berberine exerts nephroprotective effect against cisplatininduced kidney damage through inhibition of oxidative/ nitrosative stress, inflammation, autophagy and apoptosis. Food Chem Toxicol 2013;62:397-406.

10. Yang L, Brooks CR, Xiao S, et al. KIM-1-mediated phagocytosis reduces acute injury to the kidney. J Clin Invest 2015;125:1620-36.

11. Pabla N, Dong Z. Cisplatin nephrotoxicity: mechanisms and renoprotective strategies. Kidney Int 2008;73:9941007.

12. Liu D Q, Chen S P, Sun J, et al. Berberine protects against ischemia-reperfusion injury: A review of evidence from animal models and clinical studies. Pharmacol Res 2019;148:104385.

13. Mizushima N, Komatsu M. Autophagy: renovation of cells and tissues. Cell 2011;147:728-41.

14. Ashrafi G, Schwarz TL. The pathways of mitophagy for quality control and clearance of mitochondria. Cell Death Differ 2013;20:31-42.

15. Stallons LJ, Funk JA, Schnellmann RG. Mitochondrial Homeostasis in Acute Organ Failure. Curr Pathobiol Rep 2013;1:10.1007/s40139-013-0023-x.

16. Parikh SM, Yang Y, He L, et al. Mitochondrial function and disturbances in the septic kidney. Semin Nephrol 2015;35:108-19.

17. Emma F, Montini G, Parikh SM, et al. Mitochondrial dysfunction in inherited renal disease and acute kidney injury. Nat Rev Nephrol 2016;12:267-80.

18. Mizushima N, Yoshimori T. How to interpret LC3 
immunoblotting. Autophagy 2007;3:542-5.

19. Kabeya Y, Mizushima N, Ueno T, et al. LC3, a mammalian homologue of yeast Apg8p, is localized in autophagosome membranes after processing. EMBO J 2000;19:5720-8.

20. Xie Z, Klionsky DJ. Autophagosome formation: core machinery and adaptations. Nat Cell Biol 2007;9:1102-9.

21. Pankiv S, Clausen TH, Lamark T, et al. p62/SQSTM1 binds directly to Atg8/LC3 to facilitate degradation of ubiquitinated protein aggregates by autophagy. J Biol Chem 2007;282:24131-45.

22. Deng Y, Xu J, Zhang X, et al. Berberine attenuates autophagy in adipocytes by targeting BECN1. Autophagy 2014;10:1776-86.

23. Fan X, Wang J, Hou J, et al. Berberine alleviates oxLDL induced inflammatory factors by up-regulation of autophagy via $\mathrm{AMPK} / \mathrm{m}$ TOR signaling pathway. J Transl Med 2015;13:92.

24. Qin X, Jiang M, Zhao Y, et al. Berberine protects against

Cite this article as: Qi J, Xue Q, Kuang L, Xie L, Luo R, Nie $\mathrm{X}$. Berberine alleviates cisplatin-induced acute kidney injury by regulating mitophagy via PINK 1/Parkin pathway. Transl Androl Urol 2020;9(4):1712-1724. doi: 10.21037/tau-20-1129 diabetic kidney disease via promoting PGC-1 $\alpha$-regulated mitochondrial energy homeostasis. Br J Pharmacol 2020;177:3646-61.

25. Zhang M, Zhang Y, Xiao D, et al. Highly bioavailable berberine formulation ameliorates diabetic nephropathy through the inhibition of glomerular mesangial matrix expansion and the activation of autophagy. Eur J Pharmacol 2020;873:172955.

26. Qin X, Zhao Y, Gong J, et al. Berberine Protects Glomerular Podocytes via Inhibiting Drp1-Mediated Mitochondrial Fission and Dysfunction. Theranostics 2019;9:1698-713.

27. Zhang X, Cheng X, Yu L, et al. MCOLN1 is a ROS sensor in lysosomes that regulates autophagy. Nat Commun 2016;7:12109.

(English Language Editor: J. Gray) 